\title{
Evaluation of some biochemical changes in Type 2 Diabetic Patients in kalar province
}

\author{
Seerwan A. Raheem \\ *Assistant Lecturer, Bio Dept, college of education, Garmian University, Kalar, Sulaimani, \\ Kurdistan Region, Iraq
}

\begin{abstract}
The purpose of the study was to observe the fasting blood glucose and lipid profile (TG and Cholesterol). The study included 35 diabetic cases and ages ranging from (36-66) years. The family history of each individual was recorded in a questionnaire form. In addition, the study included 15 non-diabetic healthy persons as control group. The cases and control group were grouped according to the age and sex. Blood samples were collected from both groups and sera were separated and used for the determination of TG, Cholesterol and fasting blood glucose were estimated manually by the use of Spectrophotometer. In the current study, the age groups (46-55) and (56-66) included the highest number of diabetic people, which represented $37.14 \%$ and $34.29 \%$ of the total 35 cases. However, the age group (36-45) included the lowest number of the diabetic people, which represents $26.57 \%$ of the total diabetic cases. Furthermore, $71.43 \%$ of diabetic were married, concerning smoking, $74.29 \%$ of among the studied cases were smokers. The results of the biochemical analysis showed a significantly high level of fasting serum glucose, in diabetic cases of both sexes at both studied age groups, The results of the biochemical analysis showed a significantly of high level of total cholesterol (TC) and triglycerides (TG) diabetic cases of both sex.
\end{abstract}

Key Words: lipid profile, TG, Cholesterol, diabetic.

\section{Introduction:}

Diabetes mellitus is one of the main widespread health problems in the world and it is getting worse particularly in the developing countries, and all over the world, thus the disease constitutes a major health concern, presently, it is an incurable metabolic disorder which affects about $2.8 \%$ of the global population (Etuk, 2010).

Prevalence of diabetes mellitus is increasing worldwide, in line with lifestyle changes and population aging. In particular, the rising prevalence of diabetes is closely linked with that of 
obesity. World Health Organization (W.H.O.) estimates that at least 177 million people worldwide suffer from diabetes and this figure is likely to be more than double by the year 2030 (WHO, 2003).

In the USA, there are an estimated 23.6 million people (7.8\% of the population) with diabetes with 17.9 million being diagnosed (American diabetes Association, 2008), 90\% of whom are type 2 (Inzucchi and Sherwin, 2007). With prevalence rates doubling between 1990 and 2005, CDC (center for disease control and prevention) has characterized the increase as an epidemic (Gerberding, 2007). The complications of diabetes include all organs in the body (Vassort and Turan, 2010).

Diabetes mellitus is a disorder that affects the body's ability to make or use insulin. Insulin is a hormone produced in the pancreas that helps transport glucose (blood sugar) from the bloodstream into the cells so they can break it down and use it for fuel. People cannot live without insulin (Adam, 1997). In diabetes mellitus (DM), the disorders of carbohydrates, lipids and proteins metabolism play predominant role in diabetic complications. Hypercholesterolemia (CHOL) and hypertriglyceridemia (TG) are mostly observed and related largely to the degree of diabetic control (Patersonet al., 1991).

The more prevalent form, type 2 diabetes, accounts for more than $90 \%$ of cases (Olefsky, 2001). Type 2 diabetes usually begins as insulin resistance, a disorder in which the cells do not use insulin properly. As the need for insulin rises, the pancreas gradually loses its ability to produce it (Cohen, 2006). Dyslipidemia is one of the major cardiovascular disease (CVD) risk factors and plays an important role in the progress of atherosclerosis, the underlying pathology of CVD. The prevalence of dyslipidemia in type 2 diabetes is double with respect to the general population. These are more complex abnormalities that are caused by the interrelation among obesity and insulin resistance (Samatha, et al., 2012) Cholesterol, and triglycerides as well as 12.6 times higher probability to have hyper insulinemia. It is worth to emphasize that the fatty tissue is exclusively related to risk factors, such as the altered insulin and lipid profile, which can contribute to the development of the insulin resistance syndrome, which comprises several risk factors for the emergence of cardiovascular complications. In patients with type 2 diabetes, which is equivalent to CHD (coronary heart disease), it is most commonly characterized by elevated TG. (American Diabetes Association: 2011).

\section{Materials and methods}




\subsection{Materials}

Total Cholesterol Bio labo kit. France, Triglycerides Bio labo kit. France, Glucose Bio labo kit. ,Germany

\subsection{Study population}

This was a prospective longitudinal study conducted in kalar. The study included (35) subjects of age range 36-65 years with type 2 diabetes disease, who were attended to the hospital in kalar general hospitals during the 2015 to January 2016. Subjects were approached after the clinical diagnosis was done in the hospital; the patients were grouped according to the age, gender, marital status, smoking, Also the study were included (15) apparently healthy or non-diabetes. Subjects who matched as control group, they were selected as a healthy group for comparison.

\subsection{Methods}

Venous blood samples were obtained from both patients and healthy counter groups by sterile disposable syringe from an arm vein since these vessels are usually large, close to the skin surface, and easy to penetrate. $5 \mathrm{ml}$ of blood was obtained placed into sterile test tubes. The blood samples were collected then centrifuged for 10 minutes at $4000 \mathrm{rpm}$ at $4 \mathrm{C}$. The serum was separated at once by gel tube and the obtained serum was stored under the freezing point $-80 \mathrm{C}$ for the chemical analysis. The serum was used for determination of serum lipid profiles, and serum glucose.

\subsection{Biochemical analysis}

\subsubsection{Determination of serum glucose}

level employing glucosticks with the glucometer (Accu-Chek, Roch diagnostic GmbH, Mannheim, Germany).

\section{Reference values}

Adults $70-105 \mathrm{mg} / \mathrm{dL}$

\subsubsection{Determination of serum total cholesterol (TC)}

Serum total cholesterol is determined by the enzymatic method using commercial laboratory kit purchased from (BIOLABO - FRANCE).

Serum total cholesterol $(\mathrm{mg} / \mathrm{dl})=$ Optical density of test / Optical density of standard * Standard concentration $(200 \mathrm{mg} / \mathrm{dl})$. The optical density was read at $500 \mathrm{~nm}$ 


\section{Reference values}

$\begin{array}{ll}\text { total cholesterol } & \mathrm{mg} / \mathrm{dL} \\ \text { Recommended values } & <200 \\ \text { Low risk } & 200-239 \\ \text { High risk } & \geq 240\end{array}$

\subsubsection{Determination of serum triacylglycerol (TG)}

Enzymatic colorimetric test is used for serum TG measurement triacylglycerol kit also was purchased from (BIOLABO--FRANCE).

Serum triacylglycerol is calculated according to the following equation: France,Spectrophotometer, Shimadzu, Japan, Centrifuge, Hittch Serum triacylglycerol $(\mathrm{mg} / \mathrm{dl})=$ Optical density of test / Optical density of standard $\times$ Standard concentration $(200 \mathrm{mg} / \mathrm{dl})$. The optical density was read at $500 \mathrm{~nm}$.

\section{Reference valuesa}

$\begin{array}{ll}\text { Triglycerides } & \mathrm{mg} / \mathrm{dL} \\ \text { Recommended values } & 35-160\end{array}$

\subsection{Statistical analysis}

The data of current study was expressed as mean plus minus standard error of mean (Mean \pm S.E.M) and the SPSS (statistical package for social science) (Version 19) statistical software was used to analyze the data. Differences in mean values between 2 groups were analyzed by using a two-sample independent t-test,

\section{Results}

\section{Aging}

The current study showed the incidence of diabetes in relation to age was ranged from 36 to 66 years with the mean age (51) years. Result showed highest ratio of diabetes among adults at age group between (46-55) years, that represented about $37.14 \%$ of the enrolled patients in current study and the lowest distribution was observed in the age group between (36-45) years that represent about $26.57 \%$ of patients (Table 3.1 and Fig. 3.1). 
Table 3.1: The incidence of the DM in relation to age in both sexes

\begin{tabular}{|l|c|c|c|c|}
\hline $\begin{array}{c}\text { age groups } \\
\text { Dm .patient }\end{array}$ & $\mathbf{3 6 - 4 5}$ & $\mathbf{4 6 - 5 5}$ & $\mathbf{5 6 - 6 6}$ & total \\
\hline no.of patiens & 10 & 13 & 12 & 35 \\
\hline$\%$ of Patients & 26.57 & 37.14 & 34.29 & $100 \%$ \\
\hline
\end{tabular}

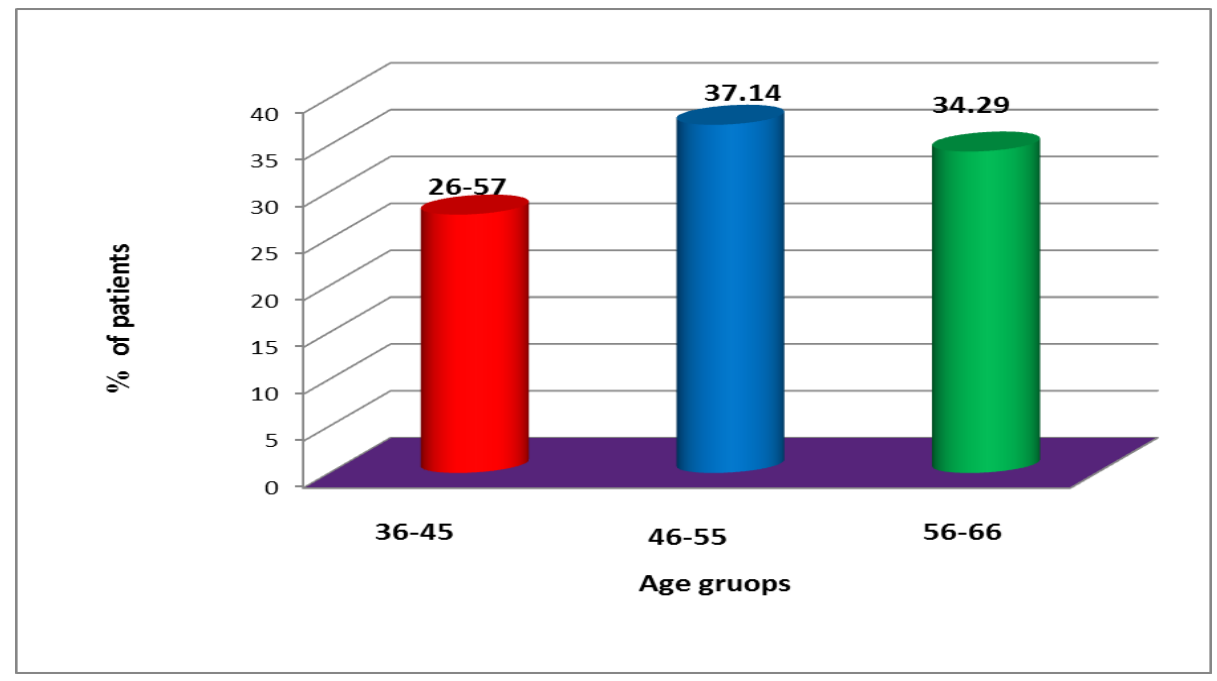

Figure 3.1: The incidence of the DM in relation to age

\section{Gender}

The results of the current study show that diabetes incidence depend on gender. Table 3.2 and figure 3.2 shows highest prevalence of diabetes in the females that represent about $(\mathbf{5 7 \%})$ of the diabetes patients, as compared with the male diabetic patients that represent about $(\mathbf{4 3} \%)$.

Table 3.2: The distribution of DM patients in correlation to gender

\begin{tabular}{|c|c|c|}
\hline Dm .patient & No & $\%$ \\
\hline Gender & 15 & $43 \%$ \\
\hline Male & 20 & $57 \%$ \\
\hline Female & 35 & $100 \%$ \\
\hline Total & & \\
\hline
\end{tabular}




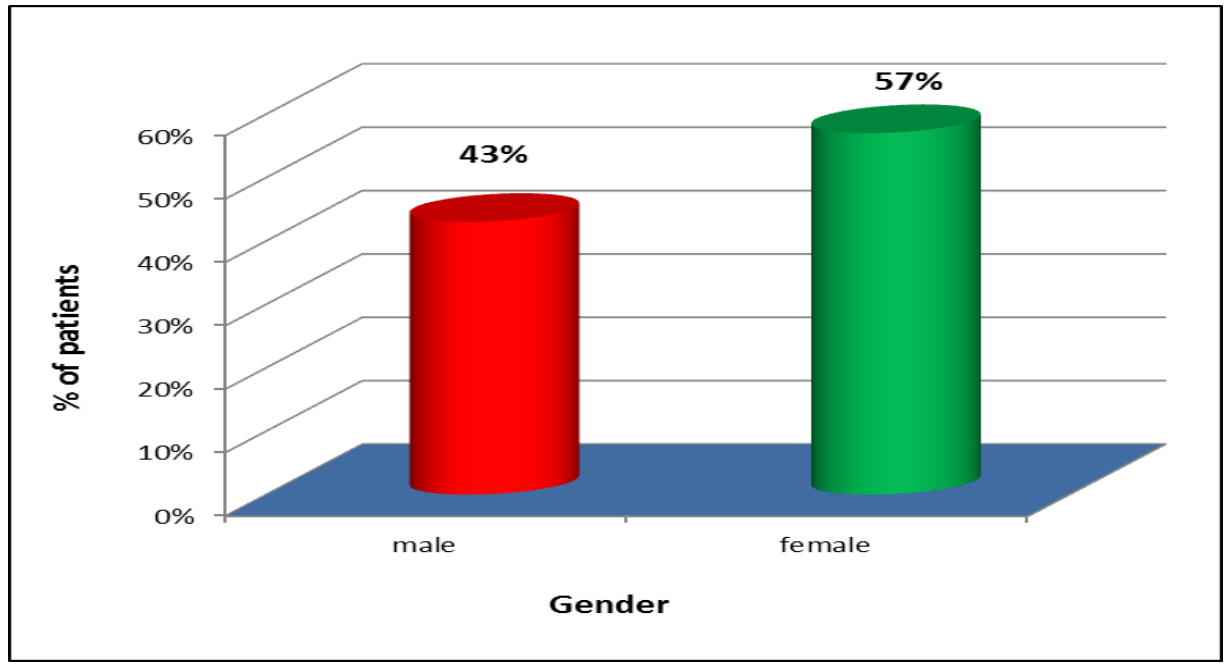

Figure 3.2: The distribution of DM patients in correlation to gender

\section{Marital status}

The results of the effect of marital status on diabetes in enrolled population show a positive correlation between diabetes and marital status (table 3.3 and figure 3.3). The result showed highest incidence of diabetes in married status that represent $(71.43 \%)$ of diabetic patients and lowest ratio was observed in single status that represent $(28.57 \%)$ of diabetes patients.

Table 3.3: The incidence of diabetes with marital status

\begin{tabular}{|c|c|c|}
\hline DM.patient & No & $\%$ \\
\hline Marital status & 25 & $71.43 \%$ \\
\hline Single & 10 & $28.57 \%$ \\
\hline Total & 35 & $100 \%$ \\
\hline
\end{tabular}

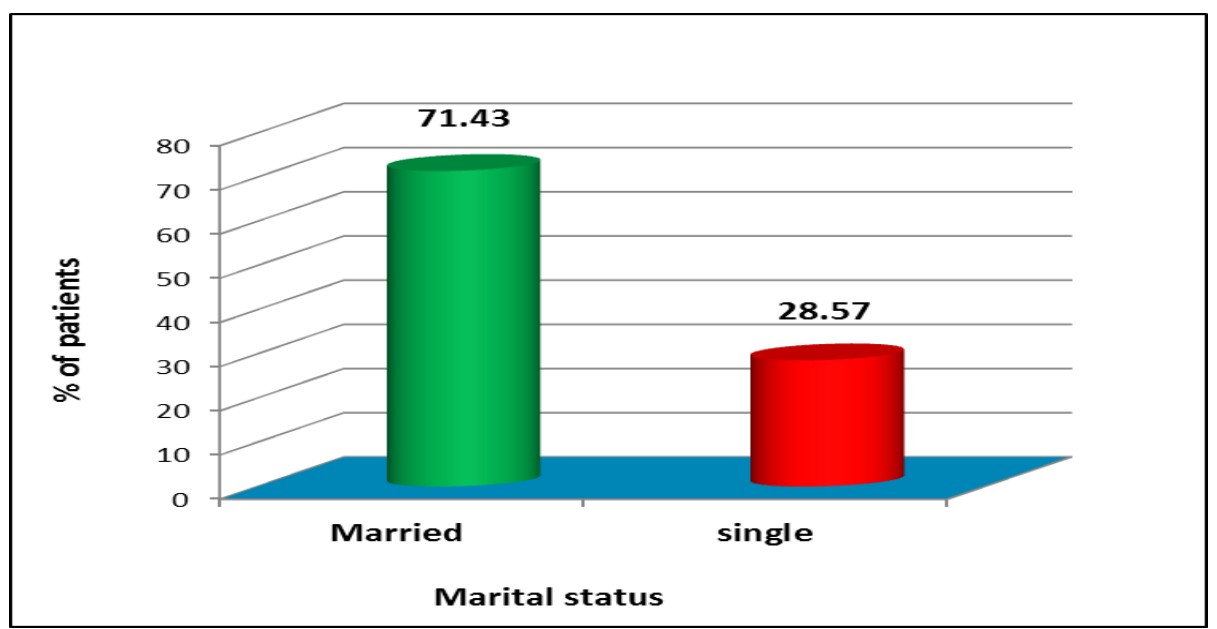

Figure 3.3: The incidence of DM in relation to marital status 


\section{Smoking cigarette}

The investigation of the relation of diabetes in relation to the smoking in the enrolled diabetic population was shown in the table 3.4 and figure 3.4. As in results showed there was an obvious relationship of diabetes to the smoking in the enrolled diabetes population, the high incidence of diabetes was seen in the smokers that represent about $(74.29 \%)$ of diabetic population whereas the non-smokers represent about $(25.71 \%)$ of diabetic population .

Table 3.4: The incidence of smoking in Diabetes patients

\begin{tabular}{|c|c|c|}
\hline Smoking & No. patients & $\%$ \\
\hline Smokers & 26 & 74.29 \\
\hline Non-Smokers & 9 & 25.71 \\
\hline Total & 35 & 100 \\
\hline
\end{tabular}

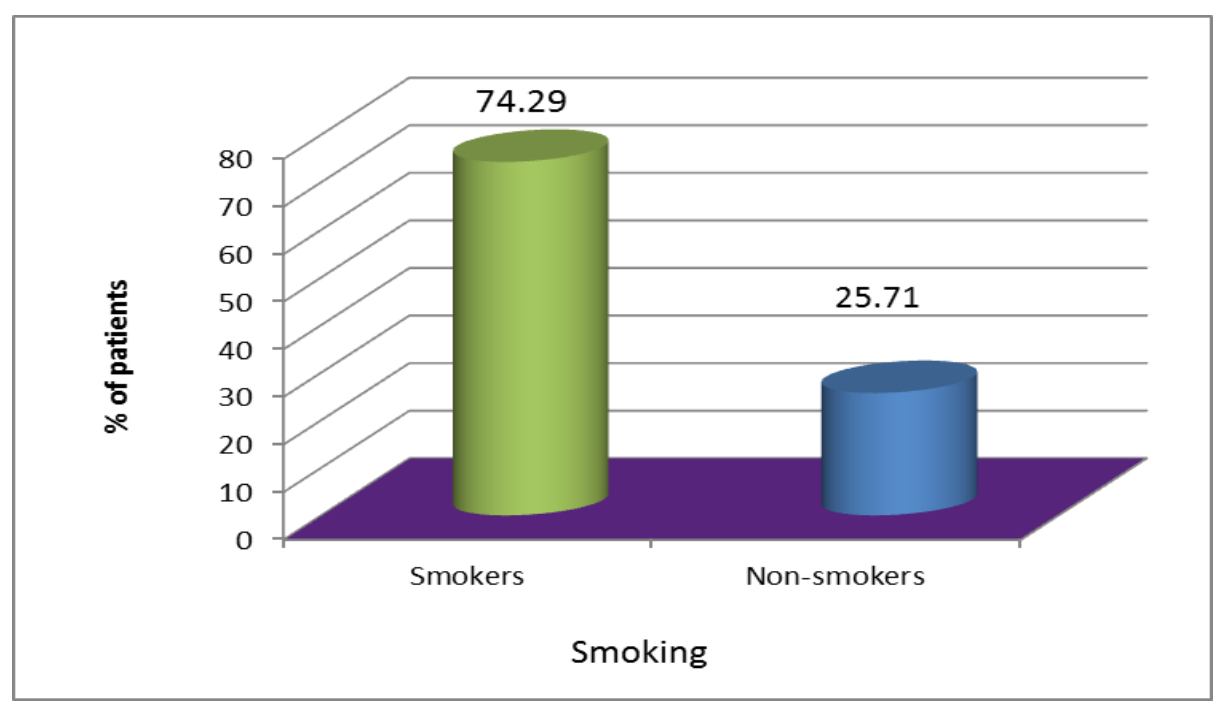

Figure 3.4: The incidence of smoking in diabetes patients

\section{Serum lipid profiles (Tc, Tg)}

The results current data show serum Glucose, Cholesterol and Triglyceride in diabetic patients. The result demonstrated a significant elevation $(\mathrm{P}<0.05)$ in a serum Glucose, TC, TG, concentrations in patients with diabetes when compared with the healthy control subjects, (Table 3.5, Figure 3.5, Figure 3.6 and Figure 3.7). 
Table 3.5: The glucose and Serum lipid profiles (Tc, $\mathrm{Tg}$ ) in Diabetic patients

\begin{tabular}{|c|c|c|c|}
\hline Group & Control mean \pm S.E. & Patients mean \pm S.E & $\begin{array}{c}\text { Statistical } \\
\text { evaluation }\end{array}$ \\
\hline parameters & $90.26 \pm 2.78$ & $201.42 \pm 10.97$ & $\mathrm{P}<0.05$ \\
\hline $\begin{array}{c}\text { Cholesterol } \\
(\mathrm{mg} / \mathrm{dl})\end{array}$ & $136.73 \pm 4.41$ & $206.22 \pm 8.00$ & $\mathrm{P}<0.05$ \\
\hline $\begin{array}{c}\text { Triglyceride } \\
(\mathrm{mg} / \mathrm{dl})\end{array}$ & $103.73 \pm 3.71$ & $171.45 \pm 19.54$ & $\mathrm{P}<0.05$ \\
\hline
\end{tabular}

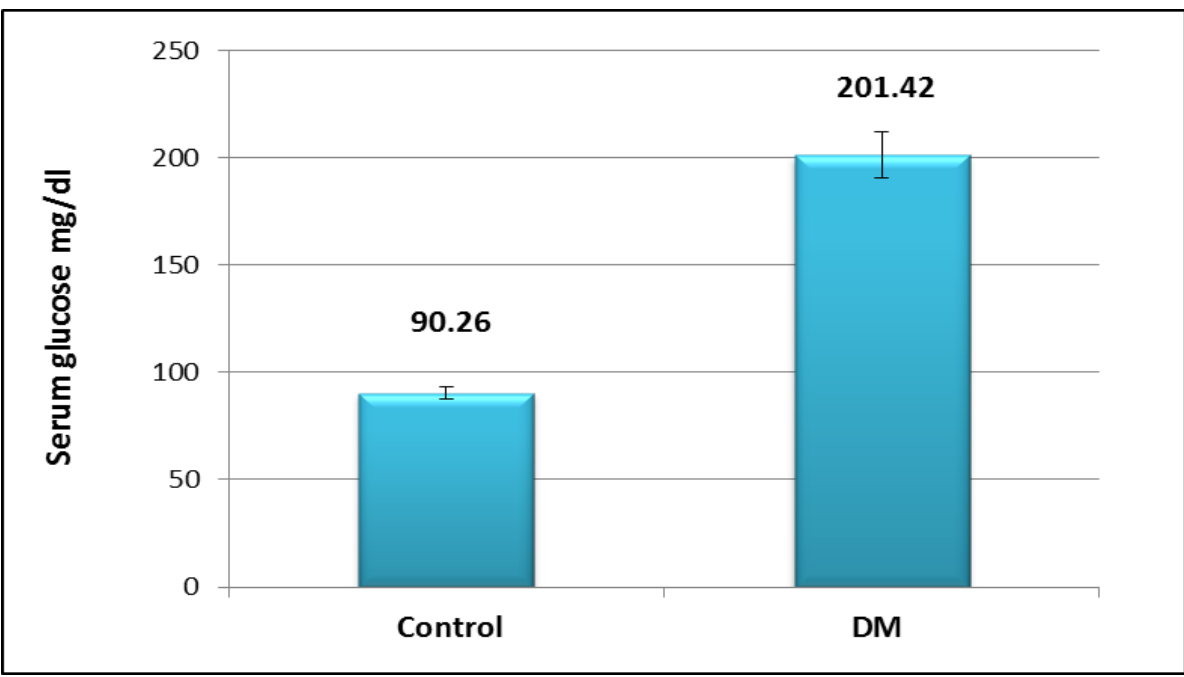

Figure 3.5: Serum glucose in Diabetic patients

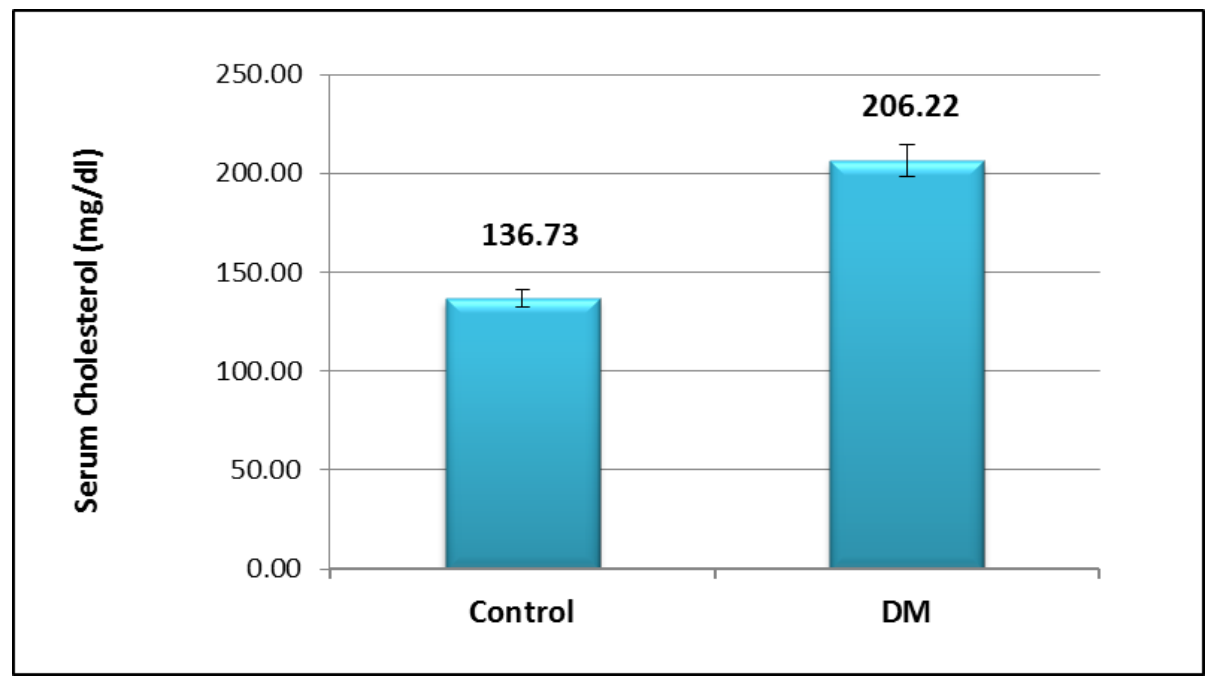

Figure 3.6: Serum Cholesterol in Diabetic patients 


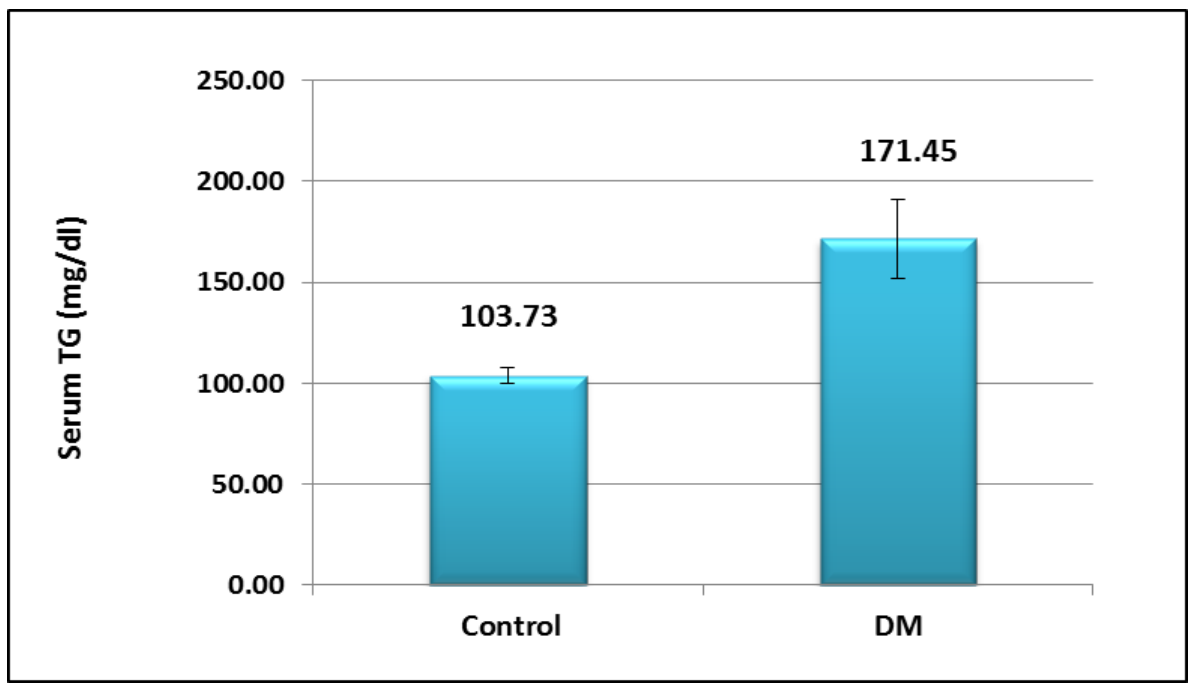

Figure 3.7: Serum Triglyceride in Diabetic patients

\section{4-Discussion}

Our finding that prevalence of diabetes increased with age is consistent with studies in other countries (Harris, 1995). Age is known to be an important determinant of diabetes since blood glucose concentrations tend to rise with age (West, 1978).

Regarding sex, while this study found a higher proportion of Female (57\%) among patients with diabetes. studies in the United States found a higher ratio of females (42\% males, $58 \%$ females) with diabetes (Harris,1995). The high proportion of females in this study may be due to the nature of population including to this hospital in that more of them seek medical attention than men under favour of having more free time because most of them were housewives

The result Found that the, Married groups had a higher prevalence of diabetes than the other marital status groups, while the single group had a low prevalence. While studies reported that Marital status has not been described previously as a risk factor for diabetes. And the single group were found to have the highest risk of diabetes (Choi and Shi, 2001)

Smoking has not been reported as a risk factor for diabetes but for diabetes associated complications such as heart diseases, Smoking predisposes people with diabetes to heart disease, peripheral vascular disease, and lower extremity amputations (Health Canada (1999). The high incidence of diabetes was seen in the smokers that represent about (74.29\%) of diabetes population whereas the non-smokers represent about $(25.71 \%)$ of diabetic population. While some studies indicated a small adverse effect of smoking on diabetes in men. This effect was not observed in women. For both men and women, former daily smokers had a higher prevalence of diabetes than daily smokers and non-smokers (Choi and Shi, 2001). 
Lipid abnormalities are common in diabetics and frequently seen in type-2 diabetics. Dyslipidaemias make diabetics prone to develop CVD. In addition, other complications of atherosclerosis (Haffner, 1998). The reason for difference in serum cholesterol values may be due to difference in the dietary habits of the people at kalar .the present study (TC) was increased significantly in diabetic patients. This result was consistent with other studies (Ramchandra et al., 2012). The present study ( $\mathrm{Tg}$ ) was increased significantly in diabetic patients. This result was consistent with other studies (Abou-Seif, and Youssef, 2004). The elevated triglyceride levels may be due to impaired activity of lipoprotein lipase (Kes,2001).

\section{Conclusions and Recommendations}

It is concluded from the results of the present study that type 2 diabetics were either and dyslipidaemia was very common. Results strongly suggest that further investigations should relate the effects of dyslipidaemia and abnormalities of insulin resistance in type 2 diabetics. suggesting that ethnic-specific strategies and guidelines on risk assessment and prevention of CVD due to dyslipidemia are required. Also the diabetic patients had a higher prevalence of high serum cholesterol, high triacylglycerol than the controls, indicating that diabetic patients were more prone to cardiovascular diseases.

\section{References}

Abou-Seif, M. A., and Youssef, A. A. (2004) "Evaluation of some biochemical changes in diabetic patients". Clinica Chimica Acta, 346(2), 161-170.

Adam, P. (1997) "Evaluation and management of diabetes insipidus", American family physician, 55(6): 2146-2153.

American Diabetes Association (2008): "Total Prevalence of Diabetes and Pre-diabetes". url =http://www.diabetes.org/diabetes-statistics/prevalence.

American Diabetes Association,(2011) "Diagnosis and Classification of Diabetes Mellitus ", diabetic care, 34 (suppl. 1): S63-S65.

Centers for Disease Control and Prevention (CDC), (2005): diabetes. National Diabetes Fact Sheet, general information. CDC Division of Diabetes Translation Public Inquiries/Publications, US.

Choi . B. C. and K. F. Shi (2001) " Risk factors for diabetes mellitus by age and sex: results of the National Population Health Survey" Diabetologia 44: 1221 \pm 1231

Cohen P., (2006)" The 20th century struggle to decipher insulin signaling". Nat Rev Mol Cell Biol; 7: 867-873. 
Etuk, E.U. (2010) "Animals models for studying diabetes mellitus", Agriculture and Biology Journal of North America 1(2): 130-134.

Gerberding J L., (2007) "Diabetes, Atlanta Centres for Disease Control".

Haffner, S.M. (1998) "Management of dyslipidemia in adults with diabetes". Diabetes Care, 21: $160-78$.

Harris, M.I, (1995) "Diabetes in America. In: National Diabe- tes Data Group. 2nd edn NIH Publication No. 95 \pm 1468 , National Institutes of Health, National Institute of Diabetes and Digestive and Kidney Diseases, Bethesda, pp 1 $1 \pm 13$.

Health Canada (1999)" Diabetes in Canada: National statis- tics and opportunities for improved surveillance, preven- tion, and control. Catalogue No. H49 $\pm 121 / 1999$.

Ottawa: Laboratory Centre for Disease Control, Bureau of Cardio- Respiratory Diseases and Diabetes

Inzucchi SE., and Sherwin RS., (2007)"The Prevention of Type 2 Diabetes Mellitus". Endocrinol Metab Clin N Am 34 (2205) 199-219.

Kes P. (2001) "Lipid abnormalities in CRF, nephrotic syndrome and dialysis". Acta Med Crotica; 55 (4-5): $177-186$

Olefsky J.M.,(2001)" Prospects for Research in diabetes mellitus". The Journal of American Medical Association, 285(5): 628-632.

Paterson, J, Pettegrew A, Dominiczak MH, and Small M.(1991)"Screening for hyperlipidaemia in diabetes mellitus. Relation to glycemic control. Ann Clin Biochem;28:254- 8.

Ramchandra, K., P, Ashok V Shinde, Sangita and M Patil (2012)"Lipid profile, serum malondialdehyde, superoxide dismutase in chronic kidney diseases and Type 2 diabetes mellitus" Biomedical Research 2012; 23 (2): 207-210

Samatha, P., Venkateswarlu, M., and Siva Prabodh, V. (2012). "Lipid profile levels in type 2 diabetes mellitus from the tribal population of Adilaba in Andhra Pradesh, India". Journal of clinical and Diagnostic Research , 6(4), 590-592.

Vassort, G., and B.Turan, (2010) "Protective role of antioxidants in diabetic induced cardiac dysfunction ", Cardiovascular Toxicology 10 (2): 73-86.

West, K.M, (1978)" Epidemiology of diabetes and its vascular lesions" Elsevier Biomedical Press, New York

World Health Organization,WHO., (2003): Non communicable disease prevention and health promotion: Facts related to chronic disease; Fact sheet - diabetes, Geneva, Switzerland . 